\title{
Celiac disease and HLA-DQ genotype: Diagnosis of different genetic risk profiles related to the age in Badajoz, southwestern Spain
}

\author{
María Jesús Fernández-Cavada-Pollo ${ }^{1}$, María Inmaculada Alcalá-Peña ${ }^{1}$, María Luisa Vargas-Pérez ${ }^{1}$, \\ Esther Vergara-Prieto ${ }^{1}$, Isabel Vallcorba-Gómez-del-Valle ${ }^{1}$, Josefa Melero-Ruiz ${ }^{1}$, Ana María Márquez- \\ Armenteros ${ }^{2}$, José Alejandro Romero-Albillos², Isidoro Narváez-Rodríguez ${ }^{3}$, José Juan Fernández-de- \\ Mera $^{4}$ and Cristina González-Roiz ${ }^{1}$
}

\begin{abstract}
Departments of ${ }^{1}$ Immunology and Genetics, ${ }^{2}$ Pediatrics. Unit of Pediatric Gastroenterology, ${ }^{3}$ Gastroenterology, and ${ }^{4}$ Pathology. Complejo Hospitalario Universitario de Badajoz. Badajoz, Spain
\end{abstract}

\begin{abstract}
Background and objectives: celiac disease is associated with the HLA class II alleles: DQA $1 * 05-D Q B 1 * 02$ and $D Q B 1 * 0302$. The genetic risk for celiac disease may depend on the presence or absence of such alleles, their combination or number of copies. This study aimed to establish the differences in HLA genotypes between celiac patients diagnosed during childhood and adulthood, and between patients and healthy controls, and to determine the risk of disease in each genotypic category.

Methods: we classified 350 celiac patients at time of diagnosis and 218 controls into 14 categories according to their HLA genotype, based on the presence or absence of risk alleles.

Results: we found statistically significant differences between the genotype frequencies of celiac patients diagnosed as being children and adults. DQA1*05 (x 1 copy), DQB1*02 (x 1 copy), $D Q B 1 * 0302$ (x 0 copies) was the most frequent genotype in individuals diagnosed in childhood, whereas DQA1*05 (x 1 copy), $D Q B 1 * 02$ (x 2 copies), DQB1*0302 (x 0 copies) was the most frequent in adults. The risk for disease in each genotypic category in celiac children and adults turned out to be different. The presence of $D Q B 1 * 0302$ did not increase risk in children, but did in adults.

Conclusion: in our celiac population, we found a different genetic pattern according to age of diagnosis. That could suggest that the pathogenic mechanism of the disease is not exactly the same in both age groups, which could somehow determine clinical presentation of the disease, its epidemiology, coexisting diseases, and complications.
\end{abstract}

Key words: HLA-DQ. Celiac disease. Autoimmunity.

Received: $12-04-2013$

Accepted: 11-09-2013

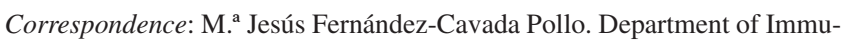
nology and Genetics. Complejo Hospitalario Universitario de Badajoz. Avda. Elvas, s/n. 06006 Badajoz, Spain

e-mail: mjfernandezcavada@yahoo.es

\section{INTRODUCTION}

Celiac disease (CD) is defined by ESPGHAN as "a systemic disorder mediated by the immune system and triggered by gluten and related prolamines in genetically susceptible individuals. It is characterized by the presence of a combination of gluten dependent clinical manifestations, specific antibodies, HLA molecules DQ2 or DQ8 and enteropathy" (1). Serological screening studies have revealed that $\mathrm{CD}$ is common, with a prevalence of about $1 \%$ of the population worldwide (2). Besides CD, there are cases of gluten reaction in which neither allergic (IgE-mediated) nor autoimmune mechanisms are involved. These are generally defined as gluten sensitivity (GS); the gastrointestinal symptoms in GS may resemble those associated with CD but it is not accompanied by the concurrence of specific celiac-related antibodies (3).

CD can appear at any age of life, although with different clinical spectrum. In children, the classic presentation with intestinal features (diarrhea with bulky, foul-smelling, floating stools due to steatorrhea and flatulence) is more common than in adults, in which non-classic extra intestinal features (anemia, osteoporosis, neurological disturbances, etc.) are more frequent (2).

In last decades, the small intestinal biopsy with typical pathological lesions was considered the gold-standard to diagnosis of $\mathrm{CD}(4,5)$. Nowadays the diagnosis of $\mathrm{CD}$, according to the new ESPGHAN criteria (published in 2012), is based on: gluten-dependent symptoms, HLA typing (presence of HLA-DQ2 and/or DQ8), CD-specific antibodies lev-

Fernández-Cavada-Pollo MJ, Alcalá-Peña MI, Vargas-Pérez ML, Vergara-Prieto E, Vallcorba-Gómez-del-Valle I, Melero-Ruiz J, Márquez-Armenteros AM, Romero-Albillos JA, Narváez-Rodríguez I, Fernández-de-Mera JJ and González-Roiz C. Celiac disease and HLA-DQ genotype: diagnosis of different genetic risk profiles related to the age in Badajoz, southwestern Spain. Rev Esp Enferm Dig 2013;105:469-476. 
els (anti-tissue transglutaminase type 2 (anti-TG2), anti-endomysium (EMA) and anti-deaminated forms of gliadin peptides (anti-DGP), and characteristic histological changes (1). A scoring system may be applied so that a stronger finding in one item may compensate for a missing abnormality in another one, and the sum could be taken into account (1). These simple diagnostic criteria could facilitate the ascertainment of CD in clinical practice, especially in "borderline" situations detected in both children and adults (6).

$\mathrm{CD}$ has a strong association with the HLA complex, mainly with HLA-DQ alleles. Indeed, more than $90 \%$ of celiac patients carry the molecule DQ2, encoded by $D Q A 1 * 05 / D Q B 1 * 02$, whereas most of the remaining patients carry $\mathrm{DQ} 8$, encoded by $D Q A 1 * 03 / D Q B 1 * 0302$ (7). These molecules are almost necessary, but not sufficient for disease development, being present in more than $30 \%$ of Caucasian individuals (8-11); thus, non-HLA loci must also contribute to the development of $\mathrm{CD}(7,12-17)$. There is compelling evidence that CD4 T cell response to HLA-DQ ( 2 or 8)-bound gluten-derived peptides (modified by tissue transglutaminase) are the primary pathogenic mechanism to disease (18).

It has been reported that the different HLA gene dosage influences the development of the disease (19). To our knowledge, differences in the association between HLA genotypes and CD diagnosed during childhood or adulthood have not been systematically examined; so, the objective of our study was to establish in our geographic area (southwestern Spain) the most frequent HLA genotypes associated with $\mathrm{CD}$ and to determine the risk for disease for each genotype depending on the age of diagnosis.

\section{MATERIALS AND METHODS}

\section{Study design}

This was a case/control study that compared CD patients (subclassified in children and adults based on the age of diagnosis) with individuals who did not have CD (controls). The goal was to retrospectively evaluate the associations between $\mathrm{CD}$, and age of diagnosis, and expression of different HLA-DQ genotypes.

\section{Patients selection}

Patients who had undergone intestinal biopsy and serologic and genetic tests for CD from 2001 to 2012 were retrospectively identified from Immunology Laboratory databases (histocompatibility, autoimmunity and cytometry). The group consisted of 355 consecutive patients with biopsy-confirmed CD (gold standard until 2012), according to the revised criteria of EPSGHAN from 1990 (5) and NASPGHAN from 2005 (4). It included 141 adults (> 14 years old, ranged from 15 to 76 years with a median age of 34 years) and 214 children (ranged from 0 to 14 years with a median age of 4 years), according to the age of diagnosis. These patients were derived from Primary Care, and Gastroenterology and Pediatrics Departments (from Badajoz, southwestern Spain).

The diagnostic tests were carried out according to a diagnostic algorithm agreed by the Departments of Immunology, Pediatrics and Gastroenterology: Serological tests -anti-TG2 antibodies, anti-gliadin (AGA) antibodies, and anti-endomysium (EMA) antibodies- were done in those patients with high clinical CD suspicion (diarrhea, flatulence, weight loss, anemia, iron deficiency, anorexia, failure to thrive, small stature, and associated diseases). In patients with $\operatorname{IgA}$ deficiency, specific antibodies of $\operatorname{IgG}$ isotype were tested. If results were positive, HLA typing was performed and, with all data, the clinicians selected the patients to perform intestinal biopsy and confirm the diagnosis of CD (20).

To avoid loss of seronegative CD cases, we also reviewed the files of Pathology and Cytometry laboratories. We did not detect any patients with negative serology and biopsy characteristic of CD: Marsh III, II and I (with increased of TCR $\gamma \delta$ and decreased of CD3(-) CD56(+) intraepithelial lymphocytes, detected by Flow Cytometry, FACScan, Beckton Dickinson, USA).

In this group, clinical, histological, and laboratory data (CD-specific antibodies, HLA-DQ typing and intra epithelial immunophenotyping), collected from medical records and laboratory databases, were irreversibly anonymized to be used in the study.

\section{Control group}

For this analysis, we included the HLA-DQ typing of 218 unrelated healthy individuals from the bone marrow donors' database, with no known autoimmune disease at the time of entry into the database, and from the same geographic area. They were between 18 and 55 years old.

The data were irreversibly anonymized to be used in the study.

This study was part of Social-Health Research Project (ref: SCSS0712) approved by the appropriate Hospital Committee.

\section{Classification of study groups}

We classified both patients and controls into 14 categories based on their genotypes, established according to the presence or absence of risk alleles, as described in table I.

\section{Serological tests}

$\operatorname{Ig} \mathrm{A} / \operatorname{IgG}$ EMA were detected by indirect immunofluorescence microscopy on rhesus monkey esophagus sub- 
Table I. Genotype categories

\begin{tabular}{|c|c|c|c|c|}
\hline & $D Q A 1 * 05$ & $D Q B 1 * 02$ & $D Q B 1 * 0302$ & Descriptive \\
\hline I & 0 & 0 & 0 & No copies \\
\hline$\|$ & 0 & 0 & 1 & 1 copy of $D Q B 1 * 0302$ \\
\hline III & 1 & 0 & 0 & 1 copy of $D Q A 1 * 05$ \\
\hline IV & 0 & 1 & 0 & 1 copy of $D Q B 1 * 02$ \\
\hline V & 1 & 1 & 0 & 1 copy of $D Q A 1 * 05$ and 1 copy of $D Q B 1 * 02$ \\
\hline $\mathrm{VI}$ & 1 & 0 & 1 & 1 copy of $D Q A 1 * 05$ and 1 copy of $D Q B 1 * 0302$ \\
\hline VII & 0 & 1 & 1 & 1 copy of $D Q B 1 * 02$ and 1 copy of $D Q B 1 * 0302$ \\
\hline VIII & 1 & 1 & 1 & 1 copy of $D Q A 1 * 05,1$ copy of $D Q B 1 * 02$ and 1 copy of $D Q B 1 * 0302$ \\
\hline IX & 0 & 2 & 0 & 2 copies of $D Q B 1 * 02$ \\
\hline$x$ & 0 & 0 & 2 & 2 copies of $D Q B 1 * 0302$ \\
\hline $\mathrm{XI}$ & 1 & 2 & 0 & 1 copy of $D Q A 1 * 05$ and 2 copies of $D Q B 1 * 02$ \\
\hline XII & 2 & 1 & 0 & 2 copies of $D Q A 1 * 05$ and 1 copy of $D Q B 1 * 02$ \\
\hline XIII & 2 & 0 & 0 & 2 copies of $D Q A 1 * 05$ \\
\hline XIV & 2 & 2 & 0 & 2 copies of $D Q A 1 * 05$ and 2 copies of $D Q B 1 * 02$ \\
\hline
\end{tabular}

The categories were established according to the number of copies of the risk alleles. We indicated as DQ2 positive, individuals carrying both alleles $D Q A 1 * 05$ and $D Q B 1 * 02$. Individuals with allele $D Q B 1 * 0302$ are indicated as $D Q 8$ positive.

strate (Medica, California, USA). An EMA(+) result was defined by the presence of the characteristic pattern of fluorescence at a dilution of $\geq 1 / 5$.

IgA/IgG anti-TG2 antibodies and $\operatorname{Ig} \mathrm{A} / \mathrm{IgG}$ AGA antibodies were determined by enzyme-immuno-assay (ORGENTEC, Mainz, Germany) from 2001 to 2008, and fluoro-immuno-assay (EliA, Thermofisher, Freiburg, Germany) from 2008; with human recombinant transglutaminase and native gliadin as antigens, respectively. Anti-TG2 antibodies level greater than $4.5 \mathrm{U} / \mathrm{mL}$ and AGA antibodies level greater than $10 \mathrm{U} / \mathrm{mL}$ were considered positive in both types of assays (according to the values recommended by the manufacturer, together with the verification tests developed in our laboratory).

The methods of different manufactures were fully comparable in terms of qualitative results (positive/negative), as we evaluated with a quality control group and external quality controls.

Serum total IgA was measured by nephelometry (Siemens, Munich, Germany) in all CD patients to screen for $\operatorname{IgA}$ deficiency. IgA deficiency was defined as a total serum IgA of less than $0.07 \mathrm{~g} / \mathrm{L}$.

\section{HLA genotyping}

Celiac and non-celiac individuals were typed for all $D Q A 1$ and $D Q B 1$ genes. This was carried out using sequence-specific oligonucleotide-polymerase chain reaction (SSO-PCR) (Gene Probe, Stamford, USA; Dynal Ltd., Bromborough, UK) and sequence-specific primer-polymerase chain reac- tion (SSP-PCR) (Invitrogen, Life technologies Ltd., Paisley, UK; Dynal Ltd., Bromborough, UK). Both methods were fully comparable in terms of results.

\section{Statistical methods}

The $\chi^{2}$ test was applied to compare the frequencies in different categories in a $2 \times 2$ contingency table, corrected with the Fisher exact test when sample sizes were small. The proportions were considered significantly different when $p<0.05$.

Disease risks were expressed as 1:n where " $n$ " was the number of individuals among which one patient was present. Considering disease prevalence in Spain of 1:118 in child population and 1:389 in adult population for each HLA category, "n" was calculated as a percentage of controls with that particular HLA status multiplied by 100 and divided by the percentage of patients with the same HLA typing $(21,22)$.

For calculation of results, all data were considered, with no missing data.

\section{Nomenclature}

We partially adopted the HLA nomenclature introduced by Megiorni et al. (22). We indicated as DQ2 positive, individuals carrying both alleles $D Q A 1 * 05$ and $D Q B 1 * 02$. Individuals with allele $D Q B 1 * 0302$ are indicated as DQ8 positive. 


\section{RESULTS}

We compared $355 \mathrm{CD}$ cases stratified by age of diagnosis (214 children, 141 adults) and 218 controls from the Immunology Laboratory databases. All of them were from southwestern Badajoz, where migratory movements are not usual (3.3 vs. $11.6 \%$ in the whole of Spain) (23). Clinical, immunological and histological data were collected at time of diagnosis, and anonymized. In the patient group, we included only those patients diagnosed of CD by the clinicians.

\section{Comparative study of risk haplotype expression in celiac patients $v s$. controls and celiac children $v s$. celiac adults (Table II)}

DQ2 were carried respectively by $89.9 \%$ of celiac patients $(92.1 \%$ children $v s .86 .5 \%$ adults) and $32.1 \%$ of controls $(p<0.001$, OR: 18.73); $98 \%$ of celiac patients were DQ2 positive and/or DQ8 positive, compared with $49.1 \%$ of healthy controls ( $p<0.001$, OR: 51.57 ), with no differences found between children and adults.

Among celiac patients, $8.2 \%$ were DQ2 negative and DQ8 positive, with differences between children and adults groups (6.5 vs. $10.6 \%)$, but with no statistical significance.

There were no significant differences in the expression of DQ8 between the control group and all celiac patients (OR: 0.58).

If we analyzed only the DQ2 negative individuals (Table II), we detected:

- In DQ2 negative patients, the percentage of adults was nearly twice the percentage in children (13.5 vs. $7.9 \%)$.
- The frequencies of cases carrying isolated DQ8 molecule were $36.1 \%$ of celiac patients vs. $14.2 \%$ of controls ( $p$ : 0.002, OR: 3.4 ), with no significant differences between celiac children and adults ( $35.3 v s .36 .8 \%)$.

- No celiac patients carried the isolated alpha 5 chain (codified by $\left.D Q A{ }^{*} 05\right)$ vs. $23 \%$ of controls that carried it.

- Among celiac patients, $33.3 \%$ (29.4\% children vs. $36.8 \%$ adults) were carriers of beta 2 chain (codified by DQB $1 * 02$ ) with DQ8, compared to $2.7 \%$ of controls $(p<0.001$, OR: 18$)$.

- No differences were found between patients and controls with the isolated beta 2 chain (11.1 vs. $16.2 \%)$.

\section{Comparative study of genotypes}

As noted in table III, we found statistically significant differences between the genotype frequencies in celiac children and adults. The most frequent genotype in children was $D Q A 1 * 05$ (x 1 copy), $D Q B 1 * 02$ (x 1 copy), $D Q B 1 * 0302$ ( 0 copies) (category V: $40.2 \%$ ), and in adults was $D Q A 1 * 05$ (x 1 copy), $D Q B 1 * 02$ (x 2 copies), $D Q B 1 * 0302$ (x 0 copies) (category XI: $32.6 \%$ ). The genotype frequencies were also different between celiac patients and the control group.

\section{Risk for disease}

We calculated the risk of developing the disease in the group of celiac children and adults, ranked by their genotype (Table IV).

Table II. Categorization of haplotypes

\begin{tabular}{|c|c|c|c|c|c|c|}
\hline Total samples & $\begin{array}{c}\% \text { controls } \\
(n=218)\end{array}$ & $\begin{array}{c}\% \text { patients } \\
(n=355)\end{array}$ & OR & $p$ & $\begin{array}{c}\% \text { children } \\
(n=214)\end{array}$ & $\begin{array}{l}\% \text { adults } \\
(n=141)\end{array}$ \\
\hline DQ2 & $32.1(70)$ & $89.9(319)$ & 18.73 & $<0.001$ & $92.1(197)$ & $86.5(122)$ \\
\hline DQ8 with/without DQ2 & $21.9(48)$ & $14.1(50)$ & 0.58 & 0.014 & $11.6(25)$ & $17.7(25)$ \\
\hline DQ8+ DQ2- & $16.9(37)$ & $8.2(29)$ & 0.43 & $<0.001$ & $6.5(14)$ & $10.6(15)$ \\
\hline DQ2(-) samples & $\begin{array}{c}\% \text { controls } \\
(n=148)\end{array}$ & $\begin{array}{c}\% \text { patients } \\
(n=36)\end{array}$ & OR & $p$ & $\begin{array}{c}\text { \% children } \\
(n=17)\end{array}$ & $\begin{array}{c}\% \text { adults } \\
(n=19)\end{array}$ \\
\hline DQ8 with/without alpha 5 chain or beta 2 chain & $25.0(37)$ & $80.6(29)$ & 12.4 & $<0.001$ & $82.4(14)$ & $78.9(15)$ \\
\hline Isolated DQ8 & $14.2(21)$ & $36.1(13)$ & 3.4 & 0.002 & $35.3(6)$ & $36.8(7)$ \\
\hline Alpha 5 chain with DQ8 & $8.1(12)$ & $11.1(4)$ & 1.4 & NS & $17.6(3)$ & $5.3(1)$ \\
\hline
\end{tabular}

DQ2: $D Q A 1 * 05$ and $D Q B 1{ }^{\star 02}$; DQ8: $D Q B 1 * 0302$; alpha 5 chain is codified by $D Q A 1 * 05$; beta 2 chain is codified by $D Q B 1 * 02 ; p<0.05$ was considered statistically significant; OR: Odds ratio; NS: No significant. 
Table III. Distribution of the genotype categories in celiac children, celiac adults, and controls

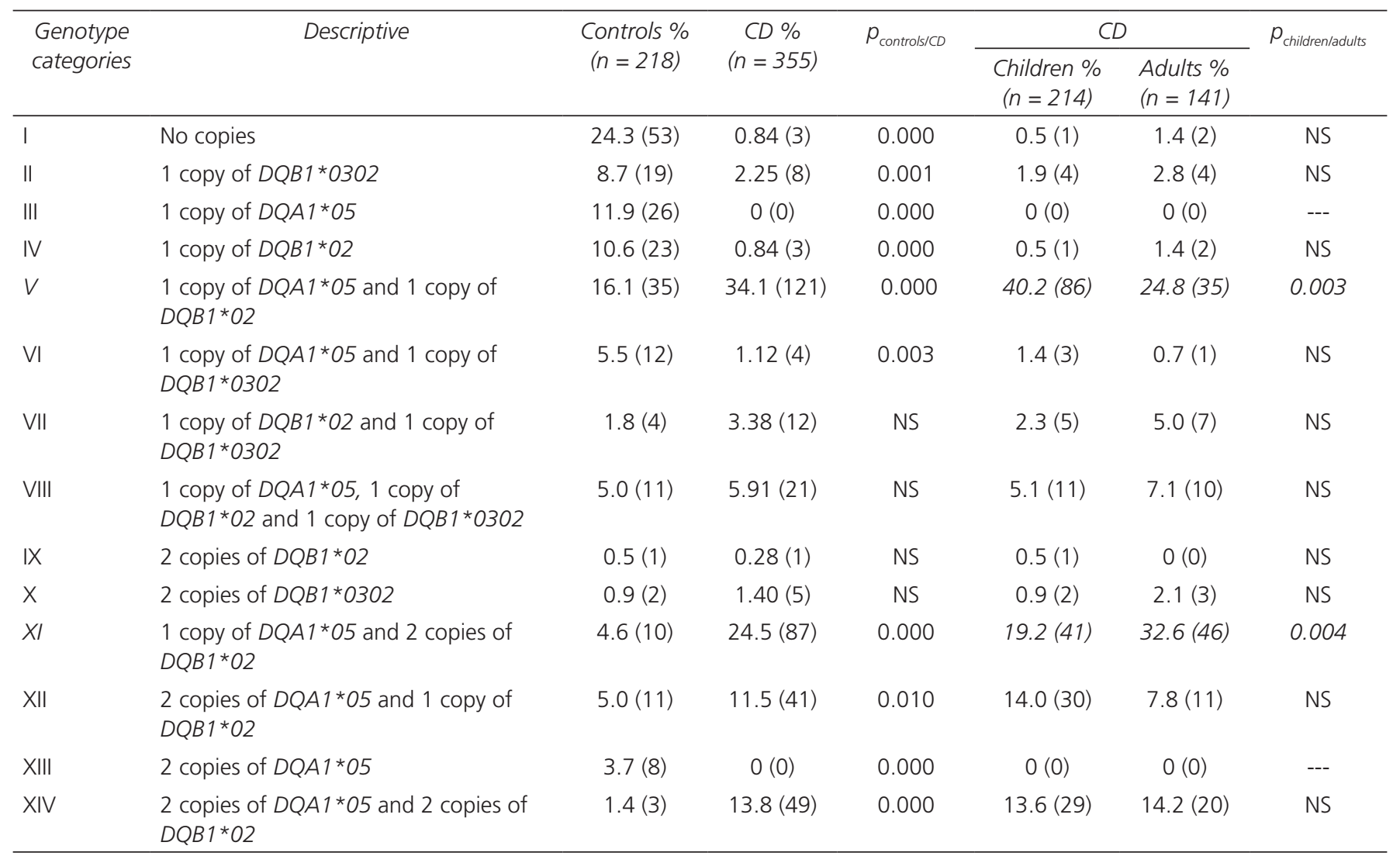

CD: Celiac disease; NS: No significance; $p<0.05$ was considered statistically significant.

Table IV. Risk gradients

\begin{tabular}{|c|c|c|c|c|c|c|}
\hline Category & Descriptive & Controls \% & $C D \%$ & $\begin{array}{l}\text { Risk in all } \\
\text { CD patients }\end{array}$ & $\begin{array}{l}\text { Risk in } C D \\
\text { children }\end{array}$ & $\begin{array}{l}\text { Risk in } C D \\
\text { adults }\end{array}$ \\
\hline XIV & 2 copies of $D Q A 1 * 05$ and 2 copies of $D Q B 1 * 02$ & 1.4 & 13.8 & $1: 10$ & $1: 10$ & $1: 10$ \\
\hline$X I$ & 1 copy of $D Q A 1 * 05$ and 2 copies of $D Q B 1 * 02$ & 4.6 & 24.5 & $1: 19$ & $1: 24$ & $1: 14$ \\
\hline XII & 2 copies of $D Q A 1 * 05$ and 1 copy of $D Q B 1 * 02$ & 5.0 & 11.5 & $1: 43$ & $1: 36$ & $1: 64$ \\
\hline V & 1 copy of $D Q A 1 * 05$ and 1 copy of $D Q B 1 * 02$ & 16.1 & 34.1 & $1: 47$ & $1: 40$ & $1: 65$ \\
\hline VII & $\begin{array}{l}1 \text { copy of } D Q B 1 * 02 \text { and } 1 \text { copy of } \\
D Q B 1 * 0302\end{array}$ & 1.8 & 3.4 & $1: 53$ & $1: 78$ & $1: 36$ \\
\hline$x$ & 2 copies of $D Q B 1 * 0302$ & 0.9 & 1.4 & $1: 64$ & $1: 100$ & $1: 43$ \\
\hline VIII & $\begin{array}{l}1 \text { copy of } D Q A 1 * 05,1 \text { copy of } D Q B 1 * 02 \text { and } 1 \\
\text { copy of } D Q B 1 * 0302\end{array}$ & 5.0 & 5.9 & $1: 85$ & $1: 98$ & $1: 70$ \\
\hline IX & 2 copies of $D Q B 1 * 02$ & 0.5 & 0.3 & $1: 178$ & $1: 100$ & 1:--- \\
\hline$\|$ & 1 copy of $D Q B 1 * 0302$ & 8.7 & 2.2 & $1: 386$ & $1: 458$ & $1: 311$ \\
\hline $\mathrm{VI}$ & 1 copy of $D Q A 1 * 05$ and 1 copy of $D Q B 1 * 0302$ & 5.5 & 1.1 & $1: 491$ & $1: 393$ & $1: 786$ \\
\hline IV & 1 copy of $D Q B 1 * 02$ & 10.6 & 0.8 & $1: 1,262$ & $1: 2,120$ & $1: 757$ \\
\hline I & No copies & 24.3 & 0.8 & $1: 2,892$ & $1: 4,860$ & $1: 1,736$ \\
\hline
\end{tabular}

HLA-DQ risk categories distribution in celiac children and adults. Risks were evaluated considering a disease prevalence of 1:118 in children and 1:389 in adults. We indicated as DQ2 positive, individuals carrying both alleles $D Q A 1 * 05$ and $D Q B 1 * 02$. Individuals with allele $D Q B 1 * 0302$ are indicated as $D Q 8$ positive. CD: Celiac disease. 
As expected, the DQ2 homozygous status (category XIV) carried the highest risk for CD (1:10 in children and adults), while the risk in category $\mathrm{V}$ (heterozygous heterodimer) was 1:40 and 1:65 in children and adults, respectively. There were differences between children and adults, depending on the genotypic category they belong to. Adults carrying one copy of $D Q B 1 * 0302$ (category II) had risk of developing the disease (1:311), while in children it was not a risk factor (1:458). In the same way, adults carrying two copies of $D Q B 1 * 0302$ had a higher risk of $C D$ than children (1:43 vs. 1:100). Furthermore, when $D Q B 1 * 0302$ and $D Q B 1 * 02$ were present (category VII), the risk of developing the disease in adults (1:36) was higher than in children (1:78). We found no celiac patients with isolated $D Q A 1 * 05$ (category III). The presence of $D Q A 1 * 05$ and $D Q B 1 * 0302$ without $D Q B 1 * 02$ (category VI) appeared to decrease the risk of developing the disease.

\section{DISCUSSION}

$\mathrm{CD}$ is a complex and multifactorial disease with environmental and genetic factors involved. Susceptibility to $\mathrm{CD}$ is related to the HLA system, mainly with the $D Q A 1 * 05$ and $D Q B 1 * 02$ alleles, which code for the DQ2 molecule, and the $D Q B 1 * 0302$ allele, that codes for the beta chain of the DD8 molecule. The higher or lower risk of developing the disease depends on the presence or absence of these alleles, their combination, and/or the number of copies (22). This disease affects individuals of any age, with different clinical presentations.

The DQ2 molecule presents gluten-derived peptides, modified by tissue transglutaminase, to $\mathrm{CD} 4+\mathrm{T}$ cells. The DQ8 molecule is capable of binding to a different set of gluten-derived peptides with different affinities that the DQ2 molecule. This finding would explain the minor association between the DQ8 molecule and the development of CD (15).

In our study area, DQ2 was present in $92.1 \%$ of the children and $86.5 \%$ of the celiac adults, in contrast with the control group (32.1\%). Our results were consistent with previous studies of celiac children in Spain (20), Northern Europe (12,24), and the United States (8). However, they did not correspond to The European Genetics Cluster on Celiac Disease, which observed less DQ2 positive patients in Southern Europe than in Northern (84.5 vs. $90.2 \%)(25,26)$.

As previous studies, we did not find significant differences in the DQ8 expression between controls and patients $(24,27)$. However, in DQ2 negative individuals, the DQ8 expression (with or without alpha 5 or beta 2 chains) were significantly higher in celiac patients than in the control group (80.6 vs. $25.0 \%$ ).

Every individual included in this study was classified into 14 categories, depending on the presence or absence of risk alleles and their combinations, and their distributions were analyzed.
As described in other studies, the most prevalent genotype in celiac patients was the $D Q A 1 * 05$ (x 1 copy), $D Q B 1 * 02$ (x 1 copy), $D Q B 1 * 0302$ (x 0 copies) (category V: $34.1 \%)(15,25,29)$.

The most frequent genotype found in celiac children was $D Q A 1 * 05$ (x 1 copy), $D Q B 1 * 02$ (x 1 copy), $D Q B 1 * 0302$ (x 0 copies) (category V: $40.2 \%$ ), like other studies (15). The most frequent genotype in celiac adults was $D Q A 1 * 05$ (x 1 copy), $D Q B 1 * 02$ (x 2 copies), $D Q B 1 * 0302$ (x 0 copies) (category XI: $32.6 \%$ ). We have not found any similar study in adult celiac patients. Paradoxically, the age of diagnosis appears to be associated with a higher genetic load. Only $13.6 \%$ of children and $14.2 \%$ of adult with CD were homozygous for DQ2 (category XIV).

It is well known that environmental factors and nonHLA alleles are involved in CD development $(7,12-17)$. Over the past three decades in western countries, the prevalence of $\mathrm{CD}$, as well as other autoimmune diseases, has had a huge increase; in CD, the use of more sensitive and specific markers, and the better understanding of the clinical forms of the disease, have increased the number of diagnosed patients. In these countries, in the same period of time, there has been a decrease of the incidence of many infections due to improvement of hygiene standards and of medical care (antibiotics, vaccination campaigns and better socio-economic conditions) (28). The "hygiene hypothesis" suggests a causal link between the increased incidence of autoimmune diseases and the decrease of usual infections, which could be explained by dysregulation of the immune system $(28,29)$. This could be favored by other environmental factors (diet, contaminants, etc.).

In our study, patients diagnosed during childhood had less genetic load than those diagnosed in adulthood. As the "hygiene hypothesis" suggests, the current environmental conditions may have altered immune system development, and these could favour the appearance of the disease at younger ages although the genetic load was less.

Then, we calculated the risk of developing CD in each genotypic category. As expected, and according to other authors $(15,25,30-34)$, the DQ2 homozygous status (category XIV) had the highest risk for CD (1:10 in children and adults patients); next in risk level were the DQ2 heterozygous categories with no DQ8 (XI, XII and V); categories with DQ8 in homozygosis or heterozygosis with $D Q B 01 * 02$ (VII, $\mathrm{X}$ and VIII) had a lower risk. When we analyzed celiac children and adults separately, the risk was different. In celiac children, the risk was similar to that described in all patients, except in DQ8 homozygous category $(\mathrm{X})$. In adults, the risk in categories with DQ8 in homozygosis or in heterozygosis with $D Q B 01 * 02$ (VII and $\mathrm{X}$ ) was higher than in the DQ2 heterozygous ones (categories XII and V). In addition, DQ8 in heterozygosis (II) conferred risk for $\mathrm{CD}$ in adults, while in children it was not a risk factor. Therefore, there could be a correlation between the presence of DQ8 and CD development in adulthood. 
The different risk pattern found between celiac children and adults could also suggest that the immune response to gluten is different in both age groups. The affinity of DQ8 to gluten is lower than that of DQ2, and each of these molecules uses different rules for the selection of epitopes for $\mathrm{T}$ cell presentation, located in different regions of gliadin (7). Exposure time to different environmental factors (i.e., tobacco) and common infections in adulthood, may lead to cross reactions with similar sequences of deaminated gluten peptides presented in the DQ8 molecule (35); this could also contribute to the different clinical presentations of the disease.

There are several studies that demonstrate that isolated $D Q B 1 * 02$ would confer a predisposition towards triggering $\mathrm{CD}(15,25,26,30,31,36-39)$, and the presence of isolated $D Q A 1 * 05$ would not $(15,22,25)$. In our study, $1 \%$ of celiac children and $1.4 \%$ of adults presented isolated $D Q B 1 * 02$ compared to healthy controls ( $11.1 \%$ of cases). Not even two copies of $D Q B 1 * 02$ increased the risk for $\mathrm{CD}$ in our study, with $0.5 \%$ of celiac children and no adults. Therefore, we could not find in our study a relationship between the presence of $D Q B 1 * 02$ and predisposition to develop the disease. Interestingly, no patient presented isolated $D Q A 1 * 05$. The presence of $D Q A 1 * 05$ does not predispose to the disease and it seems to be protective, as already described Piccini et al. (25). In fact, in our adult $\mathrm{DQ} 8+$ group, the presence of $D Q A 1 * 05$ decreased the risk of developing the disease compared to those with isolated DQ8 (1:786 vs. 1:311).

Our results corresponded to a very homogeneous ethnic group located in the southwestern of Spain, with its own environmental and genetic conditions, and where the immigration from other countries was not common (23). The exhaustive analysis of different databases from immunology and pathology laboratories and the medical record, allowed us to minimize the number of possible cases of seronegative CD. Therefore, the bias that could be provided by these cases was very low.

Our results provide the first demonstration, to our knowledge, of age-specific differences in the DQ determined susceptibility to $\mathrm{CD}$, which could suggest that the pathogenic mechanism of the disease is not exactly the same in both age groups, which could somehow determine clinical presentation of the disease, its epidemiology, coexisting diseases, complications, and association with obesity (40).

Therefore, once established genotypic differences between celiac children and adults, it would be very interesting to analyze different clinical presentations related with different risk genotypes. In conclusion, in our celiac population we found a different genetic pattern according to age of diagnosis. This genetic pattern, together with environmental and epigenetic factors, which are different between children and adults, could result in different clinical presentation of $\mathrm{CD}$. The knowledge of these factors would be useful for the design of $\mathrm{CD}$ prevention strategies.

\section{ACKNOWLEDGEMENTS}

This work was supported by: "Consejería de Sanidad y Consumo (Junta de Extremadura)" through the SocialHealth Research Project (ref: SCSS0712).

\section{REFERENCES}

1. Husby S, Koletzko S, Korponay-Szabó IR, Mearin ML, Phillips A, Shamir R, et al. European Society for Pediatric Gastroenterology, Hepatology, and Nutrition, Guidelines for the diagnosis of coeliac disease. J Pediatr Gastroenterol Nutr 2012;54:136-60.

2. Reilly NR, Green PH. Epidemiology and clinical presentations of celiac disease. Semin Immunopathol 2012;34:473-8.

3. Saponne A, Bai JC, Ciacci C, Dolinsek J, Grenn PHR, Hadjivassiliou $\mathrm{M}$, et al. Spectrum of Gluten-related disorders: consensus on the new nomenclature and classification. BMC Medicine 2012; 10:13 (http:// www.biomedcentral.com/1741-7015/10/13).

4. Hill ID, Dirks MH, Liptak GS, Colletti RB, Fasano A, Guandalini S, et al. Guideline for the diagnosis and treatment of celiac disease en children: recommendations of the North American Society for Pediatric Gastroenterology, Hepatology and Nutrition. J Pediatr Gastroenterol Nutr 2005;40:1-19.

5. Walker-Smith JA, Guandalini S, Schmitz J, Schmerling DH, Visakorpi JK. Revised criteria for diagnosis of celiac disease. Report of Working Group of European Society of Paediatric Gastroenterology and Nutrition. Arch Dis Childhood 1990;65:909-11.

6. Catassi C, Fasano A. Celiac Disease Diagnosis: Simple rules are better than complicates algorithms. Am J Med 2010;123:691-3.

7. Sollid LM, Jabri B. Celiac disease and transglutaminase 2: A model for posttranslational modification of antigens and HLA association in the pathogenesis of autoimmune disorders. Curr Opin in Immunol 2011;23:732-8

8. Fasano A, Berti I, Gerarduzzi T, Not T, Colletti RB, Drago S, et al. Prevalence of celiac disease in at risk and non at risk groups in the United States: A large multicenter study. Arch Intern Med 2003;163:286-92.

9. Koning F. Celiac disease: caught between a rock and a hard place. Gastroenterology 2005;129:1294-301.

10. Sollid LM, Lie BA. Celiac disease genetics: Current concepts and practical applications. Clin Gastroenterol Hepatol 2005;3:843-51.

11. Liu E, Rewers M, Eisenbarth GS. Genetic testing: Who should do the testing and what is the role of genetic testing in the setting of celiac disease? Gastroenterology 2005;128:S33-7.

12. Romanos J, van Diemen CC, Nolte IM, Trynka G, Zhernakova A, Fu J, et al. Analysis of HLA and non-HLA alleles can identify individuals at high risk for celiac disease. Gastroenterology 2009;137:834-40.

13. Hunt KA, Zhernakova A, Turner G, Heap GA, Franke L, Bruinenberg $\mathrm{M}$, et al. Newly identified genetic risk variants for celiac disease related to the immune response. Nat Genet 2008;40:395-402.

14. Naluai AT, Ascher H, Nilsson S, Wahlström J. Searching for genes influencing a complex disease: The case of coeliac disease. Eur J Hum Genet 2008; 16:542-53.

15. Mazzilli MC, Megiorni F, Mora B, Drago S. Genetics of coeliac disease. Autoimmun Highlights 2009; 0: 5-10.

16. Trynka G, Zhernakova A, Romanos J, Franke L, Hunt KA, Turner G, et al. Coeliac disease-associated risk variants in TNFAIP3 and REL implicate altered NF-kappaB signalling. Gut 2009;58:1078-83.

17. Plenge RM. Unlocking the pathogenesis of celiac disease. Nat Genet 2010;42: 81-2.

18. Kim CY, Quarsten H, Bergseng E, Khosla C, Sollid LM. Structural basis for HLA-DQ2 mediated presentation of gluten epitopes in celiac disease. Prov Natl Acad Sci USA 2004;101:4175-9.

19. Jabri B, Sollid LM. Mechanisms of disease: Immunopathogenesis of celiac disease. Nat Clin Pract Gastroenterol Hepatol 2006;3:516-25.

20. Vargas ML, Melero J, Fernández de Mera JJ, González C, Catalina I, Romero A. Serological and genetic markers in the diagnosis and follow-up of coeliac disease. An Pediatr (Bar) 2005;62:412-9.

21. Casellas F. Enfermedad celíaca. Med Clin (Bar) 2006;126:137-42. 
22. Megiorni F, Mora B, Bonamico M, Barbato M, Nenna R, Maiella G, et al. HLA-DQ and risk gradient for celiac disease. Hum Immunology 2009;70:55-9.

23. Fundación BBVA, (Web en línea). www.grupobbva.com. (Consulta: 03-09-2013).

24. González C, López MJ, Fernández L, Pedrera JD. Celiac disease is a good disease-HLA association model. J Pediatr Gastroenterol Nutr 2004:39:569-70.

25. Piccini B, Vascotto M, Serracca L, Luddic A, Margollicci MA, Balestri $\mathrm{P}$, et al. HLA-DQ typing in the diagnostic algorithm of celiac disease. Rev Esp Enferm Dig 2012;104:248-54.

26. Karrel K, Louka AS, Moodie SJ, Ascher H, Clot F, Greco L, et al. HLA types in celiac disease patients not carrying the DQA $1 * 05-\mathrm{DQB} 1 * 02$ (DQ2) heterodimer: Results from the European genetics cluster on celiac disease. Human Immunol 2003;64:469-77.

27. Peña L, Torres MJ, Déniz MC, Ortigosa L, Ramos JC, Calvo F, et al. Assessment of the DQ heterodimer test in the diagnosis of celiac disease in the Canary Islands (Spain). J Pediatr Gastroenterol Nutr 2003;37:604-8

28. Bach JF. The effect of infections on susceptibility to autoimmune and allergic disease. N Engl J Med 2002;346:1740-2.

29. Chatenoud L, You S, Okada H, Kuhn C, Michaud B, Bach JF. 99th Dahlem Conference on infection, inflammation and chronic inflammatory disorders. Immune therapies of type I diabetes: new opportunities based on the Hygiene Hypothesis. Clin Exp Immunol 2010;160:106-12.

30. Vermeulen BA, Hogen Esch CE, Yuksel Z, Koning F, Verduijn W, Doxiadis Il, et al. Phenotypic variance in childhood coeliac disease and the HLA-DQ/DR dose effect. Scand J Gastroenterol 2009;44:40-5.
31. Wolters VM, Wijmenga C. Genetic background of celiac disease and its clinical implications. Am J Gastroenterol 2008;103:190-5.

32. Margaritte-Jeannin P, Babron MC, Bourgey M, Louka AS, Clot F, Percopo S, et al. HLA-DQ relative risks for celiac disease in European populations: A study of the European Genetics Cluster on Coeliac Disease. Tiss Antigen 2004;63:562-7.

33. Van Heel DA, Hunt K, Greco L, Wijmenga C. Genetics in celiac disease. Best Pract Res Clin Gastroenterol 2005;19:323-39.

34. Dubois PC, van Heel DA. Translational mini-review series on the immunogenetics of gut disease: Immunogenetics of celiac disease. Clin Exp Immunol 2008;153:162-73.

35. Nieuwenhuizen WF, Pieters RH, Knippels LM, Jansen MC, Koppelman SJ. Is Candida albicans a trigger in the onset of coeliac disease? The Lancet 2003;361(9375):2152.

36. Pietzak MM, Schofield TC, McGinniss MJ, Nakamura RM. Stratifying risk for celiac disease in a large at-risk United States population by using HLA alleles. Clin Gastroenterol Hepatol 2009;7:966-71.

37. Castro-Antunes MM, Crovella S, Brandão LA, Guimaraes RL, Motta ME, Silva GA. Frecuency distribution of HLA DQ2 and DQ8 in celiac patients and first-degree relatives in Recife, norheastern Brazil. Clinics 2011;66:227-31.

38. Kagnoff MF. Celiac Disease: pathogenesis of a model immunogenetic disease. J Clin Invest 2007;117:41-9.

39. Bourgey M, Calcagno G, Tinto N, Gennarelli D, Margaritte-Jeannin P, Greco L, et al. HLA related genetic risk for coeliac disease. Gut 2007:56:1054-9.

40. Poddar U. Pediatric and adult celiac disease: similarities and differences. Indian J Gastroenterol 2013;32:283-8. 